\title{
Thermal properties of a photon gas in photonic crystals
}

\author{
Chul-Sik Kee, Sang Soon Oh, K. J. Chang, Jae-Eun Kim, and Hae Yong Park \\ Department of Physics, Korea Advanced Institute of Science and Technology, Taejon 305-701, Korea \\ Keun-Ho Lee \\ CAE, Semiconductor $R \&$ D Center, Samsung Electronics, Yongin 449-900, Korea \\ (Received 7 May 1999)
}

\begin{abstract}
We investigated the thermal properties of a photon gas in photonic crystals. Since the density of photon states in photonic crystals is modified, thermal radiation of photonic crystals is suppressed inside forbidden gaps and the heat capacity of photon gas is more enhanced than $T^{3}$ as temperature increases. The heat capacities of phonons in periodic elastic composites and magnons in periodic ferromagnetic composites are also enhanced at sufficiently low temperature. [S0163-1829(99)03040-4]
\end{abstract}

\section{INTRODUCTION}

Recently, there has been a lot of attention devoted to interesting properties of periodic dielectric composites (photonic crystals). ${ }^{1-7}$ Photonic crystals can possess frequency regions in which electromagnetic waves cannot propagate in any direction. The frequency regions are called the photonic band gaps (PBG's), anologous to the electronic band gaps for electron waves in natural crystals. This concept has been rapidly extended to other materials; metals, ferromagnetic materials, elastic materials, liquid crystals, and selfassembled materials. ${ }^{8-14}$ A defect can create an allowed frequency in a forbidden region by breaking locally the periodicity of artificial crystals such as semiconductors. However, unlike semiconducting crystals, a defect frequency can be selected by designing, in principle, the shape and the size of a local defect in artificial crystals. ${ }^{15-19}$ It has been believed that the periodic variation of the dielectric constant or refractive index can give rise to PBG's. However, it was recently demonstrated that the wave impedance plays an essential role in the formation of PBG's rather than the dielectric constant or the refractive index. ${ }^{20}$ This, especially, opens the possibilities of the application of magnetic materials to photonic crystals. Photonic crystals can be used in highefficiency semiconductor lasers and LED, waveguides, optical filters, high- $Q$ resonant cavities, efficient antennas, and frequency-selective surfaces. The density of states (DOS) of an electromagnetic wave in a photonic crystal is very much enhanced near the edges of the PBG's and disappear inside the gaps. The modification of the DOS can affect atomic spontaneous-emission rates, that is, spontaneous-emission rates are suppressed inside PBG's and enhanced near PBG edges. ${ }^{21}$ It is well known that electromagnetic thermal radiation (free-photon gas) in thermal equilibrium with a heat reservior at temperature $T$ has a blackbody radiation and the heat capacity of free-photon gas is proportional to $T^{3}$. Since the spectral energy density, i.e., the energy density per unit frequency, of photon gas depends on the DOS, the thermal properties of photon gas in photonic crystals with the modified DOS will be different from those of free-photon gases. In this paper, we investigated numerically the thermal properties of photon gas in photonic crystals.

\section{CALCULATION METHODS}

In the numerical calculation, 821 plane waves were used in order to obtain photonic band structures. ${ }^{2}$ From Maxwell's equations, the magnetic field $\mathbf{H}(\mathbf{r})$ is

$$
\nabla \times\left[\epsilon^{-1}(\mathbf{r}) \nabla \times \mathbf{H}(\mathbf{r})\right]=\frac{\omega^{2}}{c^{2}} \mathbf{H}(\mathbf{r}),
$$

where $\epsilon^{-1}(\mathbf{r})$ is the inverse of the dielectric function and $c$ the velocity of photons in vacuum. Since $\epsilon^{-1}(\mathbf{r})$ is a real and periodic function of $\mathbf{r}$, we can use Bloch's theorem to expand $\mathbf{H}(\mathbf{r})$. Because of the relation $\nabla \cdot \mathbf{H}(\mathbf{r})=0, \mathbf{H}(\mathbf{r})$ is transverse. Therefore, $\mathbf{H}(\mathbf{r})=\Sigma_{\mathbf{G}} \Sigma_{\lambda=1}^{2} h_{\mathbf{G}, \lambda} \mathbf{e}_{\lambda} e^{l(\mathbf{k}+\mathbf{G}) \cdot \mathbf{r}}$, where $\mathbf{k}$ is a wave vector in the Brillouin zone that determines the propagation direction of photon, $\mathbf{G}$ a reciprocallattice vector, and $\mathbf{e}_{\lambda}$ polarizations orthogonal to $\mathbf{k}+\mathbf{G}$. The eigenvalue equation (1) can now be expressed in the $2 \mathrm{~N}$ $\times 2 \mathrm{~N}$ matrix form

$$
\sum_{\mathbf{G}^{\prime}, \lambda^{\prime}} M_{\mathbf{G}, \mathbf{G}^{\prime}}^{\lambda, \lambda^{\prime}} h_{\mathbf{G}^{\prime}, \lambda^{\prime}}=\frac{\omega^{2}}{c^{2}} h_{\mathbf{G}, \lambda}
$$

where

$$
M_{\mathbf{G}, \mathbf{G}^{\prime}}^{\lambda, \lambda^{\prime}}=|\mathbf{K}|\left|\mathbf{K}^{\prime}\right| \epsilon^{-1}\left(\mathbf{G}-\mathbf{G}^{\prime}\right)\left[\begin{array}{cc}
\mathbf{e}_{2} \cdot \mathbf{e}_{2}^{\prime} & -\mathbf{e}_{2} \cdot \mathbf{e}_{1}^{\prime} \\
-\mathbf{e}_{1} \cdot \mathbf{e}_{2}^{\prime} & \mathbf{e}_{1} \cdot \mathbf{e}_{1}^{\prime}
\end{array}\right],
$$

where $\mathbf{K}=\mathbf{k}+\mathbf{G}, \mathbf{K}^{\prime}=\mathbf{k}+\mathbf{G}^{\prime}$, and $\epsilon^{-1}(\mathbf{G})$ is the Fourier transform of $\epsilon^{-1}(\mathbf{r})$. The linear tetrahedron method was employed in order to calculate the DOS. ${ }^{22} 256 \mathbf{k}$ points in the irreducible sector of the Brillouin zone were used in the calculations and the DOS was calculated from the first band to the 200th band.

\section{RESULTS AND DISCUSSION}

Figure 1 shows the photonic band structures, in units of $2 \pi c / a$, of a photonic diamond crystal which is constructed with dielectric spheres in air and the DOS of a photon per unit volume in arbitary units, where $a$ is the lattice constant. The calculation parameters are $f=0.34$ and $\epsilon_{s}=12.25$, where $f$ is the volume fraction of dielectric spheres and $\epsilon_{s}$ the 


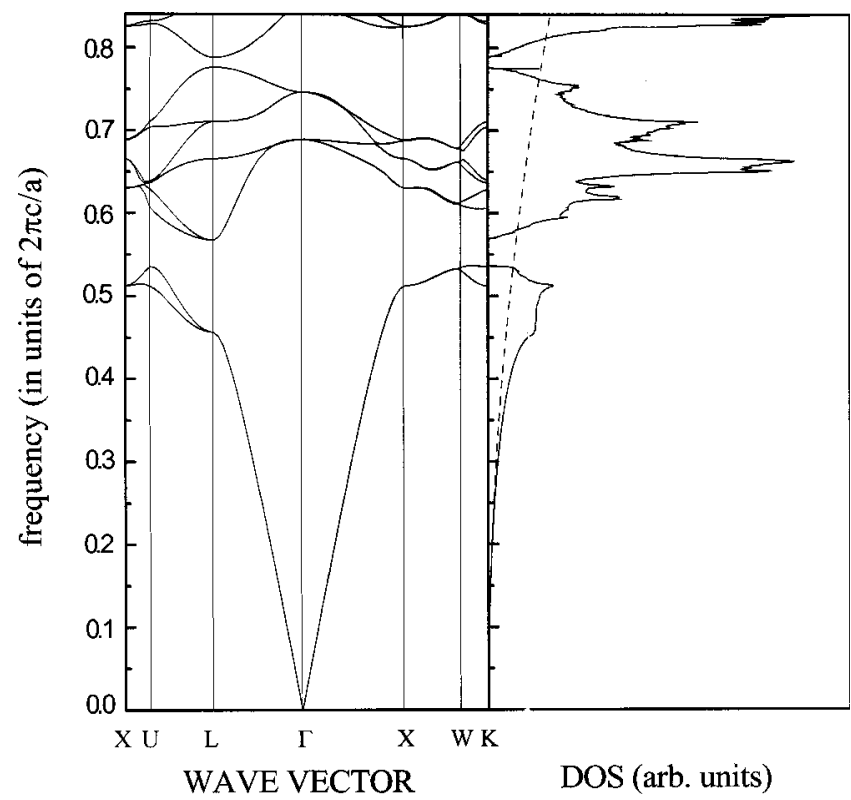

FIG. 1. Photonic band structures in a photonic diamond crystal constructed with dielectric spheres in air in units of $2 \pi c / a$ and the DOS of a photon in arbitary units. The volume fraction of the spheres is 0.34 and the dielectric constant of the spheres is 12.25. The dashed line is the DOS in a homogeneous dielectric medium with the dielectric constant 2.9. $a$ is the lattice constant and $c$ the velocity of a photon in vacuum.

dielectric constant of dielectric spheres. In a long-wavelength region $\omega a / 2 \pi c<0.3$, the DOS is proportional to $\omega^{2}$ as in a homogeneous medium. In this region, the photonic diamond crystal can be treated as a homogeneous medium with an effective dielectric constant. The effective dielectric constant of the photonic diamond crystal with the above calculation parameters is estimated to be $2.9 .^{23}$ The dashed line denotes the DOS of a photon in a homogeneous dielectric medium with the dielectric constant 2.9. The DOS was also calculated by the linear tetrahedron method. Both agree well in this region. But, the DOS is greatly modified in the wavelength region $\omega a / 2 \pi c>0.3$. The peaks in the DOS correspond to the modes whose group velocities are zero. The frequency $\omega_{\max }$ in blackbody radiation, when the spectral energy density is maximum, satisfies Wien's displacement law, i.e., $\hbar \omega_{\max }=2.82 k_{B} T$, where $k_{B}$ is the Boltzmann constant. However, it is easily expected that $\omega_{\max }$ in photonic crystals does not satisfy this relation.

Figure 2 shows the spectral energy density of photon gas in a photonic diamond crystal (solid line) in arbitary units when $k_{B} T a / h c=0.11$, where $h$ is the Planck constant. If $a$ $=1 \mu \mathrm{m}, T=1580 \mathrm{~K}$. The dashed line denotes the spectral energy density of photon gas in a homogeneous dielectric medium with the dielectric constant 2.9. So, the thermal radiation of a photonic crystal will be suppressed in forbidden gaps and enhanced in edges of the gaps. Since the total energy density of photon gas in a photonic crystal is also changed by the modification of the DOS, it is easily understood that the heat capacity of photon gas in a photonic crystal is not proportional to $T^{3}$. The heat capacity of photon gas, $c_{V}$, is

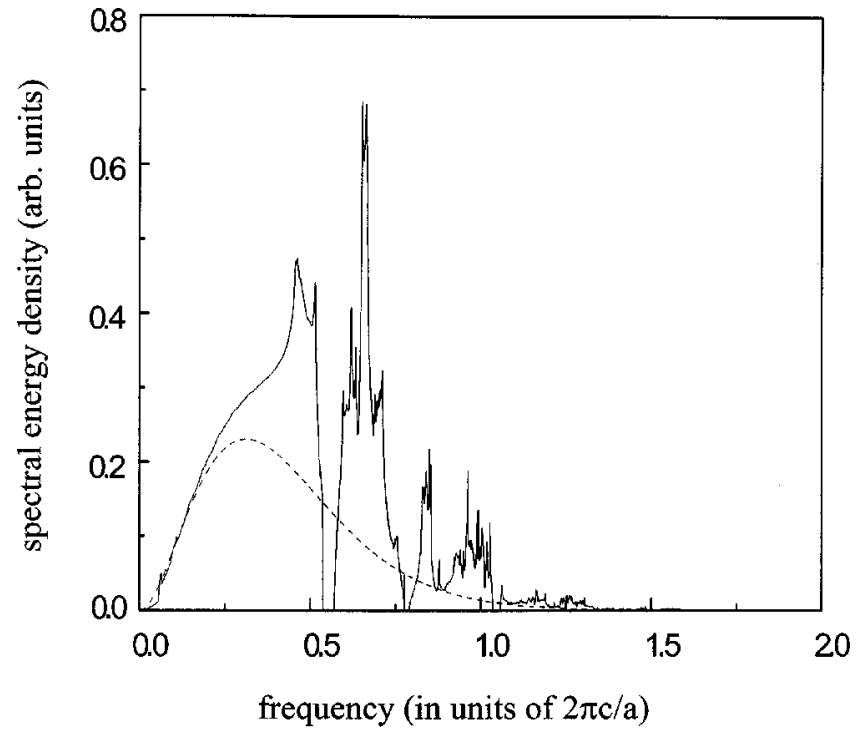

FIG. 2. Spectral energy density of photon gas in photonic diamond crystal in arbitary units when $k_{B} T a / h c=0.11$, where $k_{B}$ is the Boltzmann constant and $h$ the Planck constant. The dashed line is the spectral energy density in the homogeneous dielectric medium with the dielectric constant 2.9 .

$$
c_{V}=\frac{2 \pi}{h k_{B} T^{2}} \int_{0}^{\infty} D(\omega) \frac{(\hbar \omega)^{2} e^{\hbar \omega / k_{B} T}}{\left(e^{\hbar \omega / k_{B} T}-1\right)^{2}} d \omega,
$$

where $D(\omega)$ is the DOS of a photon per unit volume.

Figure 3 shows the heat capacity of photon gas in a photonic diamond crystal (filled circles) in arbitrary units as a function of $\left(k_{B} T a / h c\right)^{3}$. Since $k_{B} T a / h c \leqslant 0.11$, the DOS below the tenth band, where $\omega a / 2 \pi c \leqslant 1.0$, contributes mainly to the heat capacity. The upper limit of integration, 2.2, in units of $2 \pi c / a$ gives the well converged value of $c_{V}$. The heat capacity of a homogeneous medium with the dielectric constant 2.9 (open circles) is proportional to $T^{3}$. However, the heat capacity of photon gas (filled circles) in the photonic diamond crystal becomes enhanced as $T$ increases, because the contribution of the enhanced DOS to the heat capacity increases as $T$ increases.

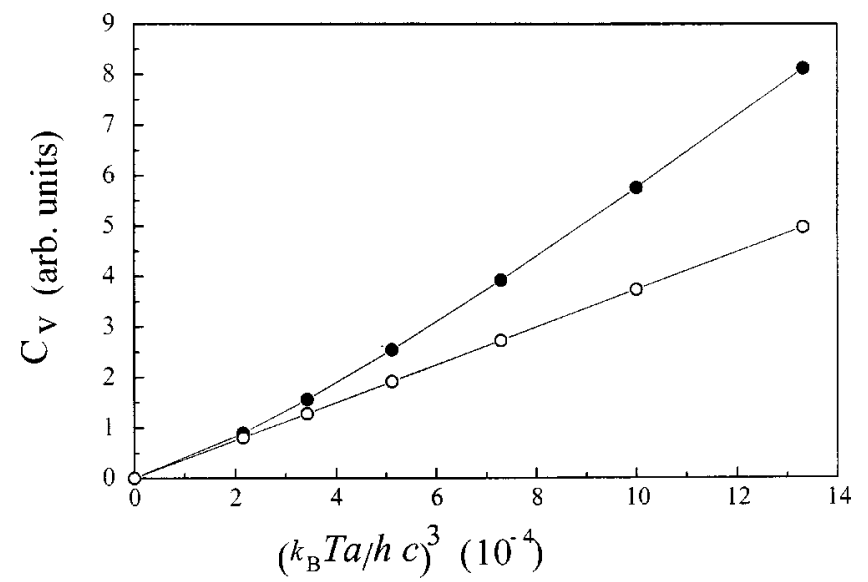

FIG. 3. Heat capacity of photon gas (filled circles) in the diamond photonic crystal in arbitary units as a function of $\left(k_{B} T a / h c\right)^{3}$. Open circles denote the heat capacity of photon gas in the homogeneous dielectric medium with the dielectric constant 2.9. 
This is a general property of the heat capacity of photongas in a photonic crystal. Although there may be computational errors due to the truncation of an infinite basis, the errors are estimated to be less than a few percent. ${ }^{24,25}$ In order to investigate the delicate dependence of the heat capacity on a higher temperature range, a great number of bases are required because the DOS of a higher frequency range should be included in the calculation. This may hit the limit of the machine. So, a more efficient method to calculate the DOS of a photon in photonic crystals should be developed.

The DOS's of phonons and magnons in periodic structures will behave very similarly to that of photons in photonic crystals, ${ }^{11,12}$ so that the heat capacity of phonons (magnons) is larger than the $T^{3}\left(T^{3 / 2}\right)$ law at low temperatures.

\section{CONCLUSION}

In conclusion, we investigated the thermal properties of photon gas in photonic crystals. Due to the modification of the density of photon states in a photonic crystal, the thermal radiation of a photonic crystal is suppressed in forbidden gaps and enhanced in the edges of the gaps. The heat capacity of photon gas in photonic crystals becomes larger than $T^{3}$ as temperature increases. The heat capacities of phonons and magnons in periodic structures are also enhanced.

\section{ACKNOWLEDGMENTS}

This work was supported in part by the Korea Science and Engineering Foundation through the Semiconductor Physics Research Center at Jeonbuk National University.
${ }^{1}$ E. Yablonovitch, Phys. Rev. Lett. 58, 2059 (1987).

${ }^{2}$ K. M. Ho, C. T. Chan, and C. M. Soukoulis, Phys. Rev. Lett. 65, 3152 (1990).

${ }^{3}$ E. Yablonovitch, T. J. Gmitter, and K. M. Leung, Phys. Rev. Lett. 67, 2295 (1991).

${ }^{4}$ S. L. McCall, P. M. Platzman, R. Dalichaouch, David Smith, and S. Schultz, Phys. Rev. Lett. 67, 2017 (1991).

${ }^{5}$ D. Cassagne, C. Jouanin, and D. Bertho, Phys. Rev. B 52, R2217 (1995).

${ }^{6}$ Cheryl M. Anderson and Konstantinos P. Giapis, Phys. Rev. Lett. 77, 2949 (1996).

${ }^{7}$ Chul-Sik Kee, Jae-Eun Kim, and Hae Yong Park, Phys. Rev. E 56, R6291 (1997).

${ }^{8}$ D. F. Sievenpiper, M. E. Sickmiller, and E. Yablonovitch, Phys. Rev. Lett. 76, 2480 (1996).

${ }^{9}$ Chul-Sik Kee, Jae-Eun Kim, and Hae Yong Park, Phys. Rev. E 57, 2327 (1998).

${ }^{10}$ D. F. Sievenpiper, E. Yablonovitch, J. N. Winn, S. Fan, P. R. Villeneuve, and J. D. Joannopoulos, Phys. Rev. Lett. 80, 2829 (1998).

${ }^{11}$ J. O. Vasseur, L. Dobrzynski, B. Djafari-Rouhani, and H. Puszkarski, Phys. Rev. B 54, 1043 (1996).

${ }^{12}$ M. S. Kushwaha, P. Halevi, L. Dobrzynski, and B. DjafariRouhani, Phys. Rev. Lett. 71, 2022 (1993).

${ }^{13}$ M. M. Sigalas and E. N. Economou, Solid State Commun. 86, 141 (1993).
${ }^{14}$ R. M. Hornreich, S. Shtrikman, and C. Sommers, Phys. Rev. E 47, 2067 (1993).

${ }^{15}$ E. Yablonovitch, T. J. Gimitter, R. D. Meade, A. M. Rappe, K. D. Brommer, and J. D. Joannopoulos, Phys. Rev. Lett. 67, 3380 (1991).

${ }^{16}$ R. D. Meade, K. D. Brommer, A. M. Rappe, and J. D. Joannopoulos, Phys. Rev. B 44, 13772 (1991).

${ }^{17}$ R. D. Meade, A. Devenyi, J. D. Joannopoulos, O. L. Alerhand, D. A. Smith, and K. Kash, J. Appl. Phys. 75, 4753 (1994).

${ }^{18}$ Chul-Sik Kee, Jae-Eun Kim, Hae Yong Park, and K. J. Chang, Phys. Rev. E 58, 7908 (1998).

${ }^{19}$ S. Fan, J. N. Winn, A. Devenyi, J. C. Chen, R. D. Meade, and J. D. Joannopoulos, J. Opt. Soc. Am. B 12, 1267 (1995).

${ }^{20}$ Chul-Sik Kee, Jae-Eun Kim, Hae Yong Park, S. J. Kim, H. C. Song, Y. S. Kwon, N. H. Myung, S. Y. Shin, and H. Lim, Phys. Rev. E 59, 4695 (1999).

${ }^{21}$ Michael D. Tocci, Michael Scalora, Mark J. Bloemer, Jonathan P. Dowling, and Charles M. Bowden, Phys. Rev. A 53, 2799 (1996).

${ }^{22}$ G. Lehmann and M. Taut, Phys. Status Solidi B 54, 409 (1972).

${ }^{23}$ S. Datta, C. T. Chan, K. M. Ho, and C. M. Soukoulis, Phys. Rev. B 48, 14936 (1993).

${ }^{24}$ H. S. Sozuer, J. W. Hans, and R. Inguva, Phys. Rev. B 45, 13962 (1992).

${ }^{25}$ H. Sami Sozuer and Joseph W. Hans, J. Opt. Soc. Am. B 10, 296 (1993). 